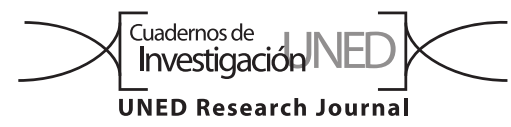

\title{
Índice de Vegetación de Diferencia Normalizada aplicado al territorio indígena agrícola de Kashaama, Venezuela
}

\author{
Barlin Orlando Olivares ${ }^{1} \&$ Miguel Armando López-Beltrán ${ }^{2}$ \\ 1. Universidad de Córdoba (UCO), Escuela Internacional de Doctorado en Agroalimentación, Programa de Doctorado en Ingeniería Agraria, \\ Alimentaria, Forestal y del Desarrollo Rural Sostenible, España; barlinolivares@gmail.com \\ 2. Universidad Autónoma de Sinaloa, Facultad de Agronomía y Desarrollo Rural, Departamento de suelo y agua, México; \\ miguel.armandolb@gmail.com
}

Recibido 09-VIII-2018 • Corregido 12-XI-2018 • Aceptado 15-XII-2018

\begin{abstract}
Normalized Difference Vegetation Index (NDVI) applied to the agricultural indigenous territory of Kashaama, Venezuela". Introduction: multi-temporal analysis of satellite images help monitor the evolution of plant communities and agricultural crops, especially in vulnerable areas. Objective: to analyze the behavior of the Normalized Difference Vegetation Index (NDVI) in the indigenous agricultural community of Kashaama, Venezuela, during the period 2012-2013. Methods: the data used was MOD13Q1 of the MODIS sensor, which contains the NDVI. Result: the average behavior of the NDVI is directly associated with the main physiographic and climatic characteristics of the region. Conclusion: the method facilitated the elaboration of maps that, together with computerized treatment of the satellite images, reliably discriminate vegetation types and their states, e.g. cultivated versus natural savannah.
\end{abstract}

Key words: remote sensing, vegetation, climate, sustainability, land.
RESUMEN: Introducción: el análisis multi-temporal de imágenes satelitales ayuda a monitorear la evolución de las comunidades de plantas y cultivos agrícolas, especialmente en áreas vulnerables. Objetivo: analizar el comportamiento del Índice de Vegetación de Diferencia Normalizada (NDVI por sus siglas en inglés) en la comunidad agrícola indígena de Kashaama, Venezuela, durante el período 2012-2013. Métodos: los datos utilizados fueron el MOD13Q1 del sensor MODIS, que incluye ese índice. Resultado: el comportamiento promedio del NDVI está directamente asociado con las principales características fisiográficas y climáticas de la región. Conclusión: el método facilitó la elaboración de mapas que, junto con el tratamiento computarizado de las imágenes de satélite, discriminan de manera confiable los tipos de vegetación y sus estados, por ejemplo, sabana cultivada versus natural.

Palabras clave: teledetección, vegetación, clima, sostenibilidad, tierra.
La vegetación representa el componente del medio físico de mayor importancia para el equilibrio del sistema ambiental, debido principalmente a que es considerado un recurso con un amplio valor intrínseco (bosques naturales, agricultura, ganadería, pastos), por otro lado, la cubierta vegetal juega un papel decisivo para el adecuado mantenimiento del resto de recursos naturales que componen el sistema (Alatorre, Miramontes-Beltrán, GarcíaPeña, Díaz-Cervantes, \& Bravo, 2014; Salinas, Martínez, \& Morales, 2017).

La mayoría de las tierras de la Mesa de Guanipa, en los Llanos Orientales venezolanos, se caracterizan por el desarrollo de operaciones de extracción de crudos pesados de las empresas petroleras en la zona, lo cual ha generado una desafectación de una cantidad considerable de hectáreas, y en consecuencia produciendo diferentes daños o perjuicios serios al medio ambiente y sus habitantes.

Particularmente en el caso de la comunidad agrícola indígena de Kashaama, se ve con preocupación la necesidad creciente de tierras para desarrollos urbanos e industriales, que pudieran cubrirse a costa de pérdida de tierra de alta capacidad para uso agrícola (Olivares, Lobo, Cortez, Rodríguez, \& Rey, 2017a; Olivares \& Franco, 2015). Adicionalmente, se reconoce que la producción agrícola en esta región del país es muy vulnerable a la ocurrencia de sequías extremas, donde las repercusiones económicas y sociales más severas de estos eventos en el territorio, las sufren las comunidades rurales y los pequeños productores, cuya capacidad de recuperación sin asistencia es limitada (Olivares, Cortez, Rodríguez, Rey, \& Lobo, 2016a; Olivares, Cortez, Lobo, Parra, Rey, \& Rodríguez, 2016b). 
Debido al tipo de bioclima seco tropical que predomina en la región de estudio, la sequía es una de las mayores amenazas de desastre natural a las que se enfrenta, cuyos efectos han sido clasificados de severos a extremadamente severos (Olivares \& Zingaretti, 2018) y acorde a las proyecciones realizadas por Trejo, Barbosa, PeñalozaMurillo, Moreno y Farías (2016a), Trejo, Barbosa, Ruiz y Peñaloza-Murillo (2016b) y Paredes, La Cruz y Guevara (2014) esta situación empeorará los próximos años.

En función a lo anterior y de acuerdo con los lineamientos del proyecto de investigación e innovación tecnológica descrito por Olivares, Cortez, Muñetones y Casana (2016c) en las zonas agrícolas vulnerables a la sequía meteorológica del sur de Anzoátegui (Olivares, Cortez, Parra, Lobo, Rodríguez, \& Rey, 2017c); los programas de seguimiento representan un punto esencial para determinar el estado de los ecosistemas de sabanas, monitorear sus cambios, y evaluar la eficacia de las tareas de gestión y conservación en las sabanas orientales venezolanas mediante el uso de herramientas como la percepción remota o teledetección y los Sistemas de Información Geográfica (SIG) para este fin.

Los SIG ofrecen la posibilidad de asociar espacialmente diferentes fuentes de datos que contengan una ubicación (datos geoespaciales). Por lo tanto, partiendo del análisis de imágenes generadas por sensores remotos, es posible obtener información de ciertas características de las coberturas vegetales a través de las diferentes bandas del espectro electromagnético y la aplicación de ecuaciones para la obtención de índices de vegetación a partir de los valores de reflectividad a distintas longitudes de onda (Hatfield, 2008; Aguilar Galindo, Fortanelli, \& Contreras, 2010).

En este orden de ideas, surge la necesidad de usar el índice de medida de la cubierta vegetal más utilizado, representado por el Índice de Vegetación de Diferencia Normalizada (NDVI-Normalized Difference Vegetation Index) (Rouse, Haas, Schell, \& Deering, 1973). Los valores de NDVI son el reflejo de la relación de la banda roja y una banda en el infrarrojo cercano (NIR). Por lo tanto, en la vegetación la banda roja se ve influenciada por la cantidad de clorofilas, mientras que la banda NIR es influenciada por el contenido de agua (Asner, 1998; Curran, 1989). Estas propiedades hacen que el NDVI se constituya en una valiosa herramienta para la evaluación de cubiertas vegetales, así como para estudiar la clasificación, dinámica vegetal y sus aspectos fenológicos (Rouse, Haas, Schell, Deering, \& Harían, 1974).

El Índice de Vegetación de Diferencia Normalizada, es un índice de gran aplicación para determinar la vegetación, ya que es eficiente para el análisis y monitoreo de las condiciones vegetativas y su dinámica en la cobertura terrestre. El NDVI es un índice que es derivado a través de la respuesta de la vegetación en relación con el espectro electromagnético, siendo expresado como lo indica la ecuación 1 (Rouse et al., 1973; Kundu, Denis, Patel, \& Dutta, 2018):

$$
N D V I=\frac{I R c-R}{I R c+R}
$$

Dónde: NDVI es el índice de vegetación cuyo resultado oscila entre -1 y 1 , cuyos valores menores a 0,1 se interpreta como suelos desnudos o cuerpos de agua, mayores a estos va en relación equivalente a la cobertura vegetal, entre más alto los valores indican la actividad fotosintética de las diversas coberturas (matorrales, bosque templado, selva y actividad agrícola). IRc representa la banda del infrarrojo cercano y $\mathrm{R}$ a la banda del rojo (Yengoh, Dent, Olsson, Tengberg, \& Tucker, 2015; Van Leeuwen, Hartield, Miranda, \& Meza, 2013).

El objetivo de este estudio fue analizar temporalmente el comportamiento del NDVI en la comunidad agrícola indígena de Kashaama, como paso para el seguimiento de la evolución de las diferentes comunidades vegetales. El desarrollo de esta metodología operativa permitiría identificar y realizar el seguimiento de las áreas afectadas en el territorio agrícola.

\section{MATERIALES Y MÉTODOS}

Definición y selección del área de evaluación: La comunidad indígena Kari'ña de Kashaama está ubicada en la parroquia Cantaura del municipio Pedro María Freites, perteneciente a la región de los Llanos Orientales venezolanos, a una distancia de $165 \mathrm{~km}$ de Barcelona, capital del Estado Anzoátegui, posee una extensión territorial de aproximadamente 5272 ha y actualmente cuenta con una población de 1392 habitantes (Olivares, Rodríguez, Cortez, Rey, \& Lobo, 2015). La delimitación del área total de la comunidad estuvo en función de los ejercicios de mapeo y a las entrevistas con los informantes claves de la comunidad (Fig. 1).

La precipitación en la comunidad de Kashaama se presenta con una estación seca bien definida de diciembre a abril, con un promedio de precipitación anual (19702000) en la región de 1119 mm (Olivares, Torrealba, \& Caraballo, 2013). La región presenta un bio-clima seco tropical según la clasificación de Holdridge, con evaporación media anual de $2626 \mathrm{~mm}$; la temperatura media anual de $26,9^{\circ} \mathrm{C}$ y la humedad relativa media anual de 78,0\% (Olivares, Parra, \& Cortez, 2017b). 


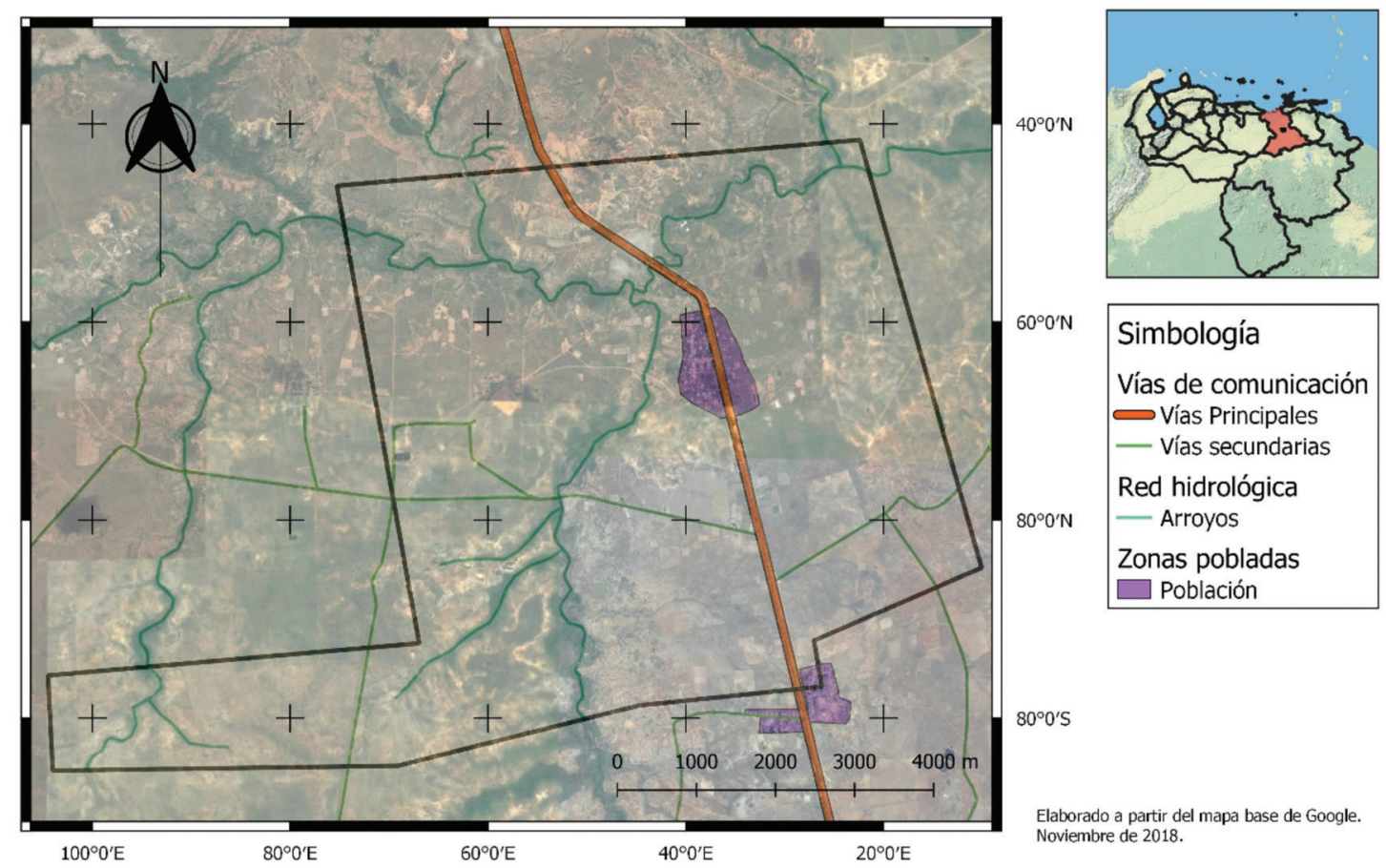

Fig. 1. Geolocalización de la comunidad indígena de Kashaama en el estado Anzoátegui, Venezuela. Fuente: elaboración propia.

Índice de Vegetación de Diferencia Normalizada (NDVI): El producto MOD13Q1 Versión 006 del sensor MODIS provee datos de índice de vegetación (NDVI y $\mathrm{EVI})$, con una resolución espacial de $250 \mathrm{~m}$ y es un producto compuesto promedio de valores de 16 días, usando el producto MxD09A1 en un periodo de 8 días en formato HDF (Herarchical Data Format) (Didan, 2015). Dichas imágenes se obtuvieron de la base de datos pública de Land Processes Distributed Active Archive Center (LPDAAC, https://lpdaac.usgs.gov/) de la Administración Nacional de la Aeronáutica y del Espacio estadounidense (NASA, por sus siglas en inglés), distribuidas por el Servicio Geológico de los EEUU (USGS).

Se extrajo las imágenes correspondientes a NDVI, por cada mes correspondieron dos imágenes, con álgebra de mapas los datos se representaron de forma mensual usando el software IDRISI Selva, Clark Labs, Clark University, Worcester MA v17.02 con una proyección UTM 20N. En este trabajo se analizó el comportamiento promedio y la tendencia sostenida del NDVI en la comunidad agrícola de Kashaama en Anzoátegui, Venezuela, para los años completos de 2012-2013, a partir de imágenes del sensor MODIS. Posteriormente se hizo un análisis de regresión de las variables precipitación mensual y valores del NDVI para los años 2012 y 2013 implementado en software estadístico R (R Development Core Team, 2008).
Ética, conflicto de intereses y declaración de financiamiento: los autores declaran haber cumplido con todos los requisitos éticos y legales pertinentes, tanto durante el estudio como en el manuscrito; que no hay conflictos de interés de ningún tipo, y que todas las fuentes financieras se detallan plena y claramente en la sección de agradecimientos. Asimismo, están de acuerdo con la versión editada final del documento. El respectivo documento legal firmado se encuentra en los archivos de la revista.

\section{RESULTADOS}

Variaciones del NDVI interanual: Los valores de NDVI se clasificaron en 5 categorías: 1-Suelo sin vegetación $(<0,2)$, 2-Poca vegetación $(0,2-0,4), 3$-Vegetación media $(0,4-0,6)$, 4-Vegetación densa $(0,6-0,8)$ y 5 -Vegetación muy densa $(>0,8)$ (Chuvieco, Deshayes, Stach, Cocero, \& Riaño, 1999).

El total de la superficie correspondiente a cada categoría del NDVI se muestra en el cuadro 1; la mayor superficie se concentra entre las categorías de poca a media vegetación con porcentajes que oscilan entre los $18,4 \%$ para algunos meses húmedos y un $82,8 \%$ para meses 
CUADRO 1

Cuantificación de la superficie (ha) según las categorías del NDVI en el área de estudio

\begin{tabular}{ccccccccccccccc} 
NDVI $^{*}$ & año & Ene. & Feb. & Mar. & Abr. & May. & Jun. & Jul. & Ago. & Sep. & Oct. & Nov. & Dic. \\
\multirow{2}{*}{1} & 2012 & 143,0 & 571,9 & 714,8 & 428,9 & 143,0 & 214,5 & 143,0 & 143,0 & 428,9 & 143,0 & 143,0 & 285,9 \\
& 2013 & 428,9 & 357,4 & 357,4 & 929,3 & 285,9 & 214,5 & 143,0 & 143,0 & 143,0 & 143,0 & 143,0 & 357,4 \\
2 & 2012 & 2430,4 & 3216,8 & 4432,0 & 4503,5 & 2501,9 & 1000,8 & 857,8 & 1000,8 & 1072,3 & 929,3 & 1644,1 & 1715,6 \\
& 2013 & 2787,9 & 3788,6 & 4289,0 & 3860,1 & 3145,3 & 2716,4 & 2144,5 & 1143,7 & 1358,2 & 2287,5 & 1787,1 & 2501,9 \\
\multirow{2}{*}{3} & 2012 & 2359,0 & 1429,7 & 285,9 & 428,9 & 2573,4 & 2573,4 & 3574,2 & 3931,6 & 3359,7 & 3645,7 & 3216,8 & 3145,3 \\
& 2013 & 2144,5 & 1143,7 & 714,8 & 643,4 & 1858,6 & 359,0 & 3002,3 & 3431,2 & 3216,8 & 2716,4 & 3431,2 & 2430,4 \\
\multirow{2}{*}{4} & 2012 & 500,4 & 214,5 & - & 71,5 & 214,5 & 1644,1 & 857,8 & 357,4 & 571,9 & 714,8 & 428,9 & 285,9 \\
& 2013 & 71,5 & 143,0 & 71,5 & - & 143,0 & 143,0 & 143,0 & 571,9 & 571,9 & 285,9 & 71,5 & 143,0 \\
\multirow{2}{*}{5} & 2012 & - & - & - & - & - & - & - & - & 0,0 & - & - & 0,0 \\
& 2013 & - & - & - & - & - & - & - & 143,0 & 143,0 & - & - & - \\
\hline
\end{tabular}

*(1: Sin vegetación; 2: Poca vegetación; 3: Vegetación media; 4: Vegetación densa; 5: Vegetación muy densa)

secos; esto es consistente si se considera que se trata de una zona de Bosque seco tropical.

Comportamiento espacial del NDVI: El comportamiento espacial del NDVI para cada pixel se puede apreciar claramente en la figura 2; durante el transcurso de los dos años bajo estudio, la vegetación densa paso del 9,2\% al 1,3\%; esto asociado claramente con las áreas de mayor cobertura vegetal localizadas en los terrenos cultivados para la campaña agrícola de 2011-2012. Por otra parte, durante el mes de mayo se observó un incremento de $11,8 \%$ de la categoría del NDVI asociado a la poca vegetación en la zona.

La figura 3 refleja en un sentido amplio, una aproximación sobre el funcionamiento del ecosistema terrestre, mayormente el comportamiento promedio del NDVI se asocia directamente con las principales características fisiográficas y climáticas presentes en la región (Peng et al., 2012). Generalmente la vegetación densa y muy densa está asociada al hábito perennifolio del ecosistema, que se diferencia de otros bosques del territorio nacional.

El análisis de regresión entre los valores de precipitación de la zona bajo estudio y los valores del NDVI $\left(R^{2}\right.$ corregido=0,2556; $p=0,0068$; error estándar $=0,0623$ ) indica una relación positiva entre las dos variables. Se observa que el comportamiento de la precipitación se refleja en el comportamiento temporal del NDVI, es decir los valores más bajos de precipitación se relaciona con los valores mínimos de NDVI (Fig. 4).

La figura 5 muestra las diferencias (ganancias y pérdidas) entre las categorías del NDVI, la mayor proporción de tierras que pasaron de poca vegetación a vegetación densa se ubica en los meses lluviosos (junio-septiembre), esto debido a que la distribución del déficit hídrico abarca el periodo desde octubre hasta mayo, con un máximo de $(212 \mathrm{~mm})$ en marzo (Olivares et al., 2015), generalmente los cultivos que se siembran después en julio corren el riesgo de sufrir déficit hídrico en la fase de floración. Solo durante la época lluviosa existe almacenamiento de agua en el suelo disponible para las plantas.

\section{DISCUSIÓN}

En general, los Llanos de Venezuela tienen una amplia connotación ecológica, por la diversidad fisionómica, estructural y funcional de las comunidades vegetales que allí se desarrollan. Esa diversidad está regulada por una cantidad de factores y procesos, entre los cuales la estacionalidad en la disponibilidad de agua en el suelo figura como uno de los condicionantes principales.

La vegetación típica de las sabanas está representada por gramíneas y algunas especies arbustivas, por su ubicación dentro del bosque seco tropical (Olivares et al., 2015). Esta vegetación es bastante pobre en cuanto a densidad, pero su composición es variada. Primeramente es de notar la escasez de vegetación alta, cuyas especies más importantes son el Chaparro (Curatella americana), Alcornoque (Bowdichia virgilioides), Tortolito (Casearia silvestris), Chaparro manteco (Byrsonima crassifolia), Merey (Anacardium occidentale). Dentro de los géneros más importantes de gramíneas que predominan en esta zona están: Trachypogon, Sporobolus, Aristida y Andropogon.

En este sentido, la geografía característica de los Llanos Orientales es la principal estrategia de la producción campesina para mantener la actividad agrícola en la zona. El productor indígena mantiene su sistema productivo, manipulando el paisaje natural, y de esa manera favorece la heterogeneidad y la diversidad biológica. La 


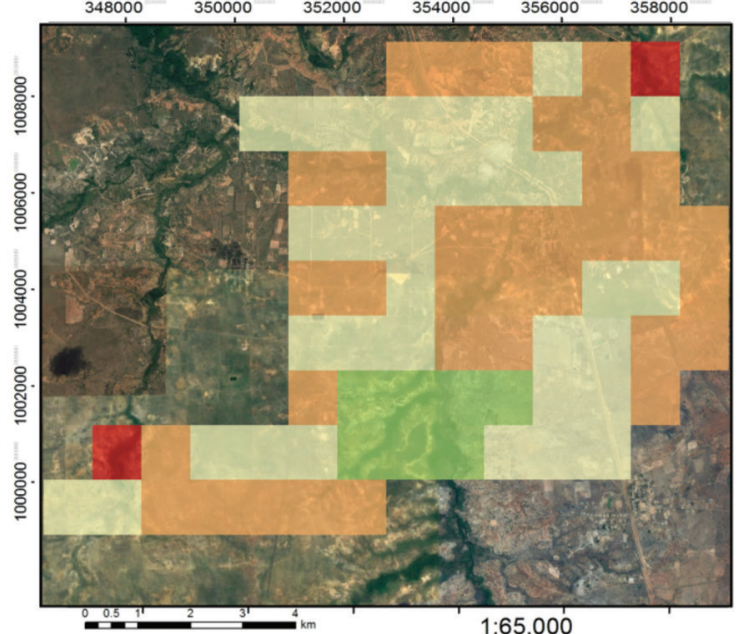

(a)

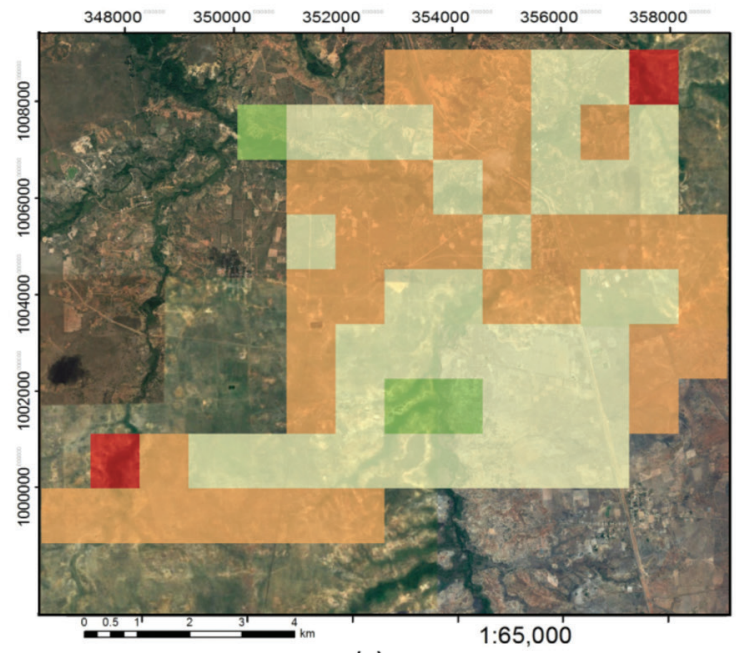

(c)

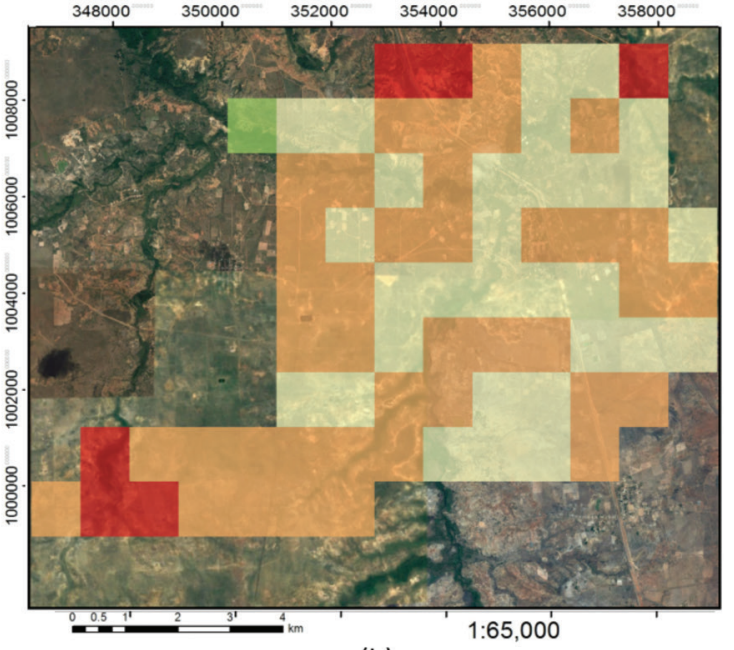

(b)

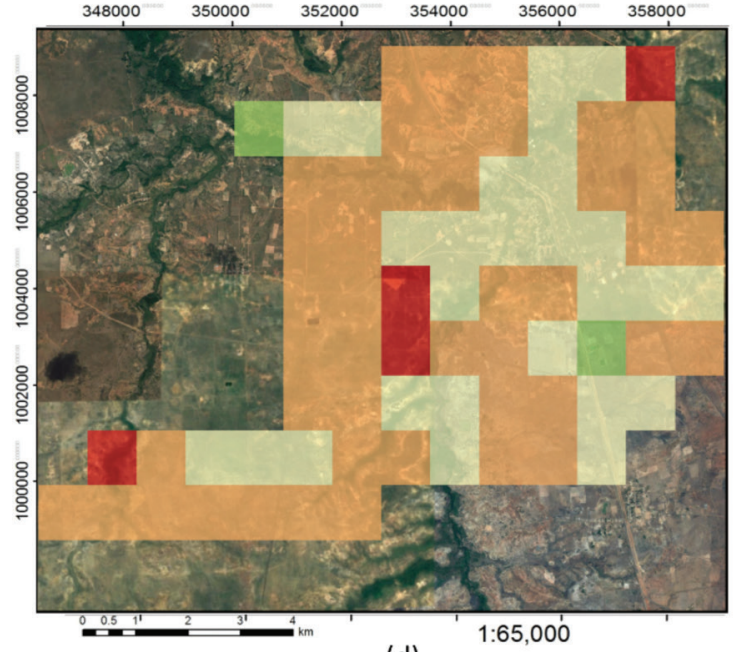

(d)
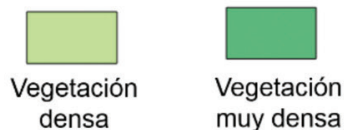

Fig. 2. Distribución espacial de las categorías del NDVI durante los meses secos (enero y mayo) en la comunidad indígena de Kashaama. (a) enero 2012; (b) enero 2013; (c) mayo 2012; (d) mayo 2013.

diversidad de los cultivos practicados por los miembros de esta comunidad indígena de Kashaama han sido ampliamente referenciados en estudios, los cuales establecen un abanico de plantas comúnmente utilizados por la etnia Kari'ña, donde solo las raíces y tubérculos tales como: Yuca amarga (Manihot esculenta Crantz), Yuca dulce (Manihot aipi Pohl), Batata (Ipomoea batatas L) junto con el plátano (Musa paradisiaca L), son considerados como básicos en su alimentación.
Por otra parte, las características biológicas de los bosques secos de esta zona del país han sido pobremente exploradas y hay poca información sobre sus características estructurales (Dezzeo, Flores, Zambrano-Martínez, Rodgers, \& Ochoa, 2008). Las semejanzas en abundancia de árboles y área basal entre el bosque ribereño siempreverde, el bosque deciduo y el semideciduo, pueden estar relacionadas con algunas características edáficas que tienden también a ser similares entre ellos, tales 


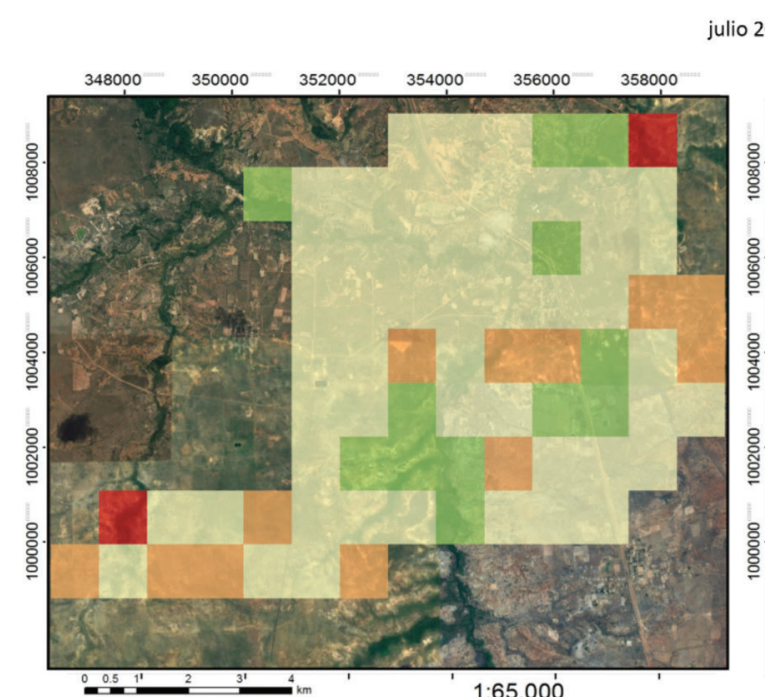

(a)

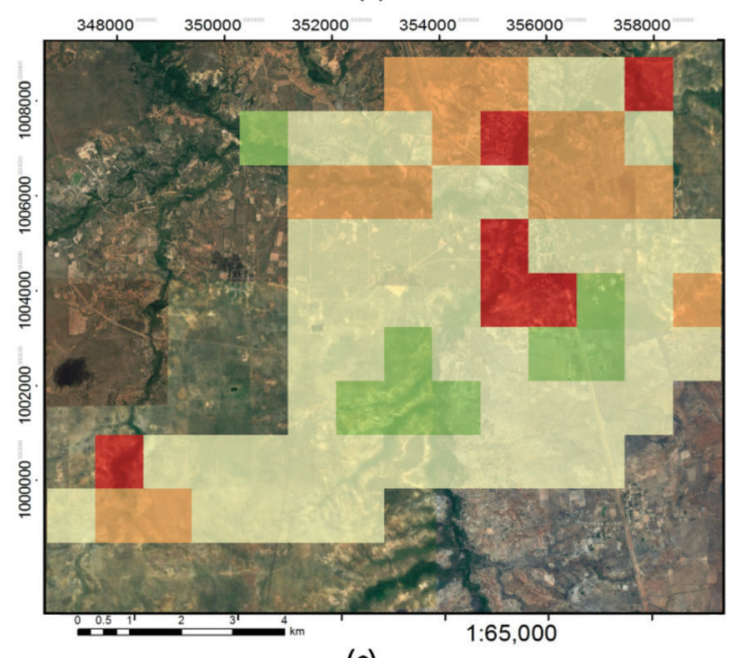

(c)

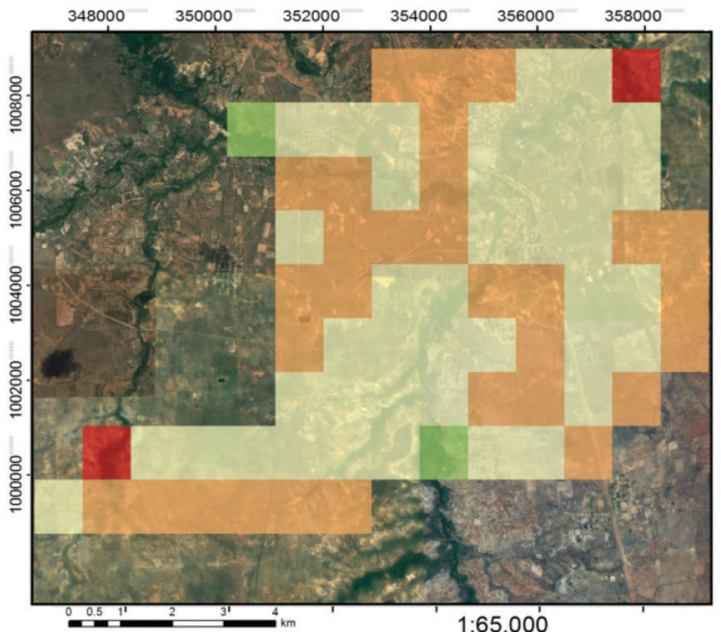

(b)

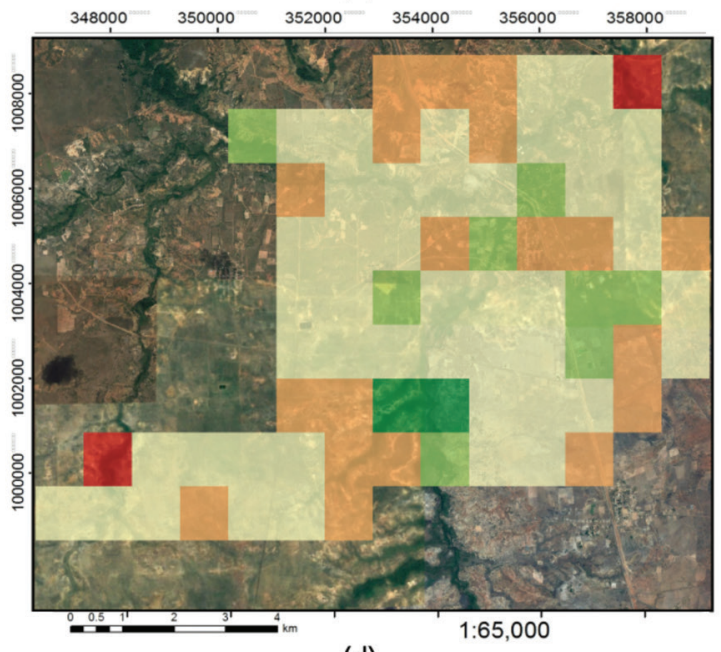

(d)
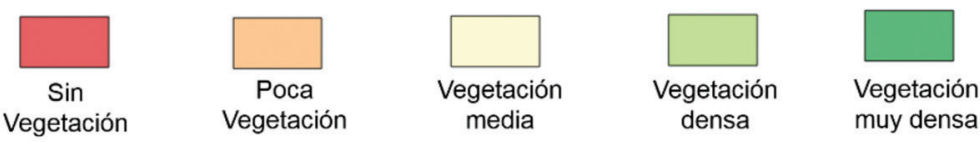

Fig. 3. Distribución espacial de las categorías del NDVI durante los meses lluviosos (julio y septiembre) en la comunidad indígena de Kashaama. (a) julio 2012; (b) julio 2013; (c) septiembre 2012; (d) septiembre 2013.

como alto contenido de arena y bajas concentraciones de $\mathrm{C}$ orgánico y $\mathrm{N}_{2}$ (Olivares, 2012; Chacón, Dezzeo, \& Flores, 2007).

De acuerdo con Erasmi, Propastin y Kappas (2009) se establece que un comportamiento cíclico anual de la vegetación, son las respuestas clásicas a un ciclo regular de pluviosidad y almacenamiento de agua en el terreno. En este sentido, los valores de NDVI mínimos se registraron entre el periodo seco de febrero a abril, mientras que los valores máximos se observaron en los meses más lluviosos comprendidos entre julio-septiembre. Por otra parte, Olivares et al. (2016b) indico que durante inicios de la época lluviosa se presentó un déficit hídrico en la zona de El Tigre que afectó el crecimiento de los cultivos tradicionales y la vegetación de sabana.

Este tipo de análisis revela ciertos cambios naturales experimentados por la vegetación a lo largo de un determinado tiempo. A los fines del seguimiento, será necesario distinguir las fluctuaciones en el verdor que resultan de oscilaciones naturales de otros procesos. Es necesario 


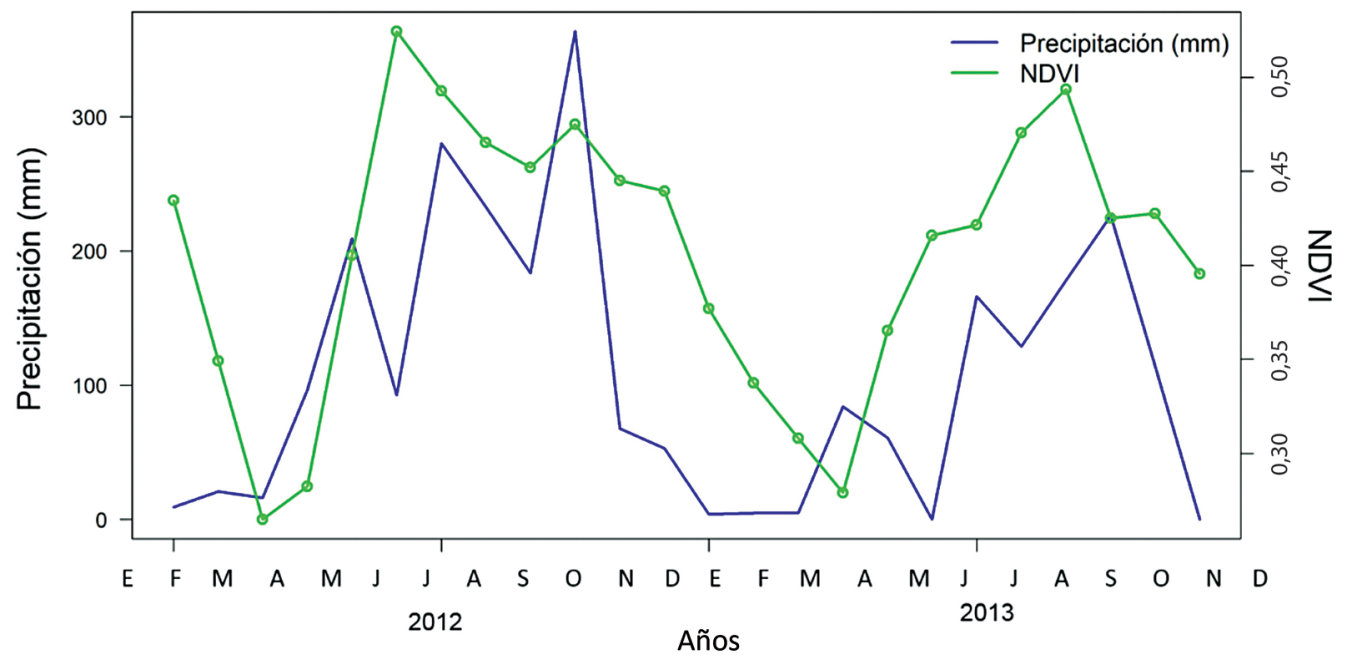

Fig. 4. Comportamiento temporal de la precipitación de la zona bajo estudio y los valores de NDVI para los años 2012 y 2013.

mencionar que existe la influencia de las anomalías climáticas que repercuten en el vigor del crecimiento. Tal es el caso de los años considerados como húmedos, asociados con el Fenómeno La Niña determinan en gran medida el incremento del valor del NDVI, mientras que los años secos, generan valores bajos del indicador de cambio (Erasmi et al., 2009; Poveda \& Salazar, 2004).

De acuerdo con las características físicas de los suelos en la Mesa de Guanipa y al régimen de precipitación en la zona, no se presentan láminas de excesos durante todo el año (Rodríguez, Cortez, Olivares, Rey, Parra, \& Lobo, 2013). Sin embargo, se presentan altos promedios de lluvia en los meses de julio y agosto, con una dispersión asociada a la variabilidad de la lluvia (Poveda \& Salazar, 2004), estableciendo cierta incertidumbre en la planificación agrícola de la zona.

Para el caso del estrés hídrico, el NDVI puede alcanzar un máximo alrededor de 0,25 a 0,33 debido a la senescencia de las hojas (valor equivalente a un campo cubierto de residuos de cosecha, o bien, a un estatus de una plantación con presencia de malezas o plagas o deficiencias en el manejo) por lo que los valores bajos de NDVI equivalen a un cultivo seco, de baja productividad y altamente estresados (Almeida, De Souza, \& Rossetto, 2006); esto se relaciona con lo reportado por Jiménez, Vargas, Salinas, Aguirre y Rodríguez (2004), donde mencionó que el factor climático y la distribución de la precipitación pluvial, junto al edafológico, limitan la productividad de diferentes cultivos de secano (Aguilar et al., 2010). Estas investigaciones indicaron que los ajustes que se obtienen entre el NDVI y la humedad del suelo desde el punto de vista espacial fueron relativamente aceptables.
La correlación lineal entre la humedad del suelo y el NDVI dependen claramente de las precipitaciones registradas durante la primavera en zonas templadas, obteniéndose mejores correlaciones, cuando la lluvia registrada en la primavera es superior a sus valores normales.

Por otra parte, la disminución de la cobertura vegetal tiene implicaciones que inciden en la disponibilidad y calidad de los bienes y servicios ambientales que ofrecen estas tierras. Entre otros, podría afectar la infiltración del agua de lluvia, ya que a pesar de ser suelos principalmente arenosos serían susceptibles al sellado y encostrado (Lozano, Lobo, \& Pla, 2002); en consecuencia, se afectaría la recarga de los mantos hídricos y el equilibrio en el ciclo del agua, considerado esto como un aspecto de importancia en este estado oriental del país, debido al potencial de los acuíferos. Las condiciones que se generan sugieren que el uso de la tierra bajo condiciones de secano debe basarse en sistemas de producción adaptados a prácticas conservacionistas, tal y como lo establecen Lobo et al. (2010).

El uso de imágenes de satélite permitió abarcar grandes extensiones de territorio para este análisis. El producto del sensor MODIS proporcionó datos de NDVI ya calculado, pero la desventaja que presentó fue la resolución espacial de $250 \mathrm{~m}$, lo que conlleva a que el pixel represente el valor con mayor información posible, es decir si la zona dispone en mayor medida de zona urbana más que vegetación, la clasificación arrojaría un área sin vegetación, como en el caso de algunos pixeles.

La metodología aplicada facilitó la elaboración de mapas de la vegetación que, en conjunto con el tratamiento informático de las imágenes satelitales, dio origen a la 


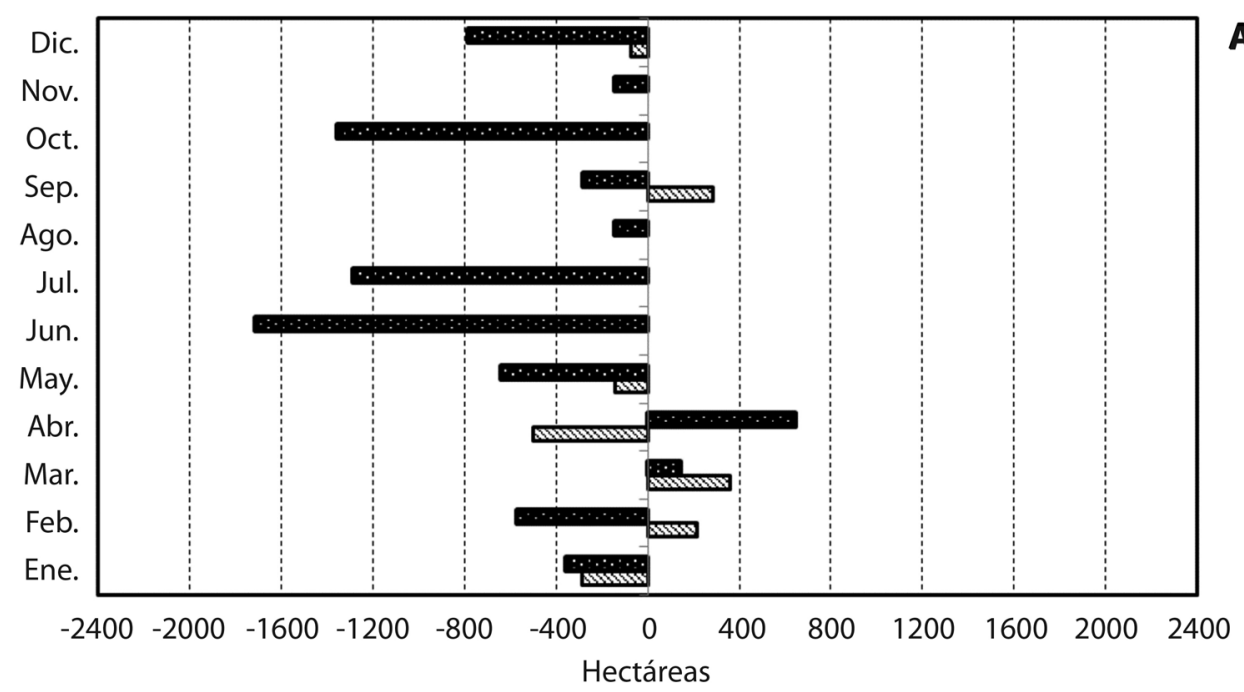

A

Poca vegetación $\mathbf{E}$ Sin vegetación

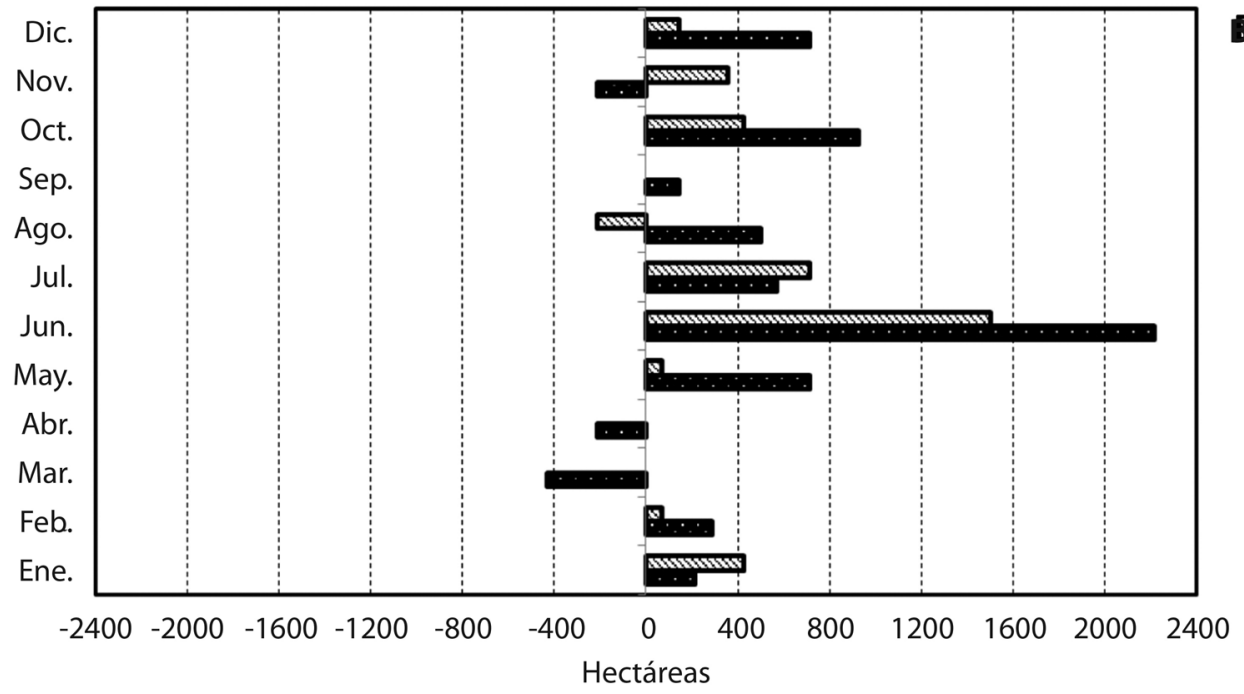

vegetación densa Vegetación media

Fig. 5. Distribución de las diferencias de superficies (2012-2013) en Kashaama: (a) categorías de poca vegetación y sin vegetación; (b) categorías de vegetación densa y media.

discriminación de la densidad de la vegetación y su estado, obteniendo la superficie cultivada o con vegetación natural de sabana para la comunidad de Kashaama.

Este estudio representa una base para seguir con la búsqueda de ciertos patrones de cambio a lo largo del tiempo que puedan ser indicadores del estado en que se encuentran los agroecosistemas de los llanos orientales venezolanos, cuyos resultados puedan ser útiles en el planteamiento estratégico para el manejo sostenible de tierras y finalmente para la toma de decisiones en el territorio.

\section{REFERENCIAS}

Aguilar, N., Galindo, G., Fortanelli, J., \& Contreras, C. (2010). Índice normalizado de vegetación en caña de azúcar en la Huasteca Potosina. Avances en Investigación Agropecuaria, 14(2), 49-65.

Alatorre, L.C., Miramontes-Beltrán, S., García-Peña, A.K., DíazCervantes, R., \& Bravo, L.C. (2014). Evolución de la dinámica vegetal mediante una serie de imágenes LANDSAT TM (1986-2011): Región central de Chihuahua, México. Cuadernos de Investigación Geográfica, 40(2), 449-476. DOI: 10.18172/cig.2510 
Almeida, T.I.R., De Souza, C.R., \& Rossetto, R. (2006). ASTER and Landsat ETM+ images applied to sugarcane yield forecast. International Journal of Remote Sensing, 27(19), 4057-4069. DOI: 10.1080/01431160600857451

Asner, G.P. (1998). Biophysical and Biochemical Sources of Variability in Canopy Reflectance. Remote Sensing of Environment, 64(3), 234-53. DOI: 10.1016/ S0034-4257(98)00014-5

Chacón, N., Dezzeo, N., \& Flores, S. (2007). Caracterización florística, estructural y edafológica de los bosques, morichales y sabanas ubicados en el área comprendida entre los ríos Sariapo y Ature, sur del Estado Anzoátegui (Informe Técnico No. 1). Caracas, Venezuela: INTEVEP.

Chuvieco E., Deshayes M., Stach N., Cocero D., \& Riaño D. (1999) Short-term fire risk: foliage moisture content estimation from satellite data. En E. Chuvieco (Ed.) Remote Sensing of Large Wildfires in the European Mediterranean Basin (pp. 17-34). Berlin, Alemania: Springer-Verlag. DOI: 10.1007/978-3-642-60164-4_3

Curran, P.J. (1989). Remote Sensing of Foliar Chemistry. Remote Sensing of Environment, 30(3), 271-78. DOI: 10.1016/0034-4257(89)90069-2

Dezzeo, N., Flores, S., Zambrano-Martínez, S., Rodgers, L., \& Ochoa, E. (2008). Estructura y composición florística de bosques secos y sabanas en los llanos orientales del Orinoco, Venezuela. Interciencia, 33(10), 733-740.

Didan, K. (2015). MOD13Q1 MODIS/Terra Vegetation Indices 16-Day L3 Global 250m SIN Grid V006 [Data set]. NASA EOSDIS LP DAAC. DOI: 10.5067/MODIS/MOD13Q1.006

Erasmi, S., Propastin, P., \& Kappas, M. (2009). Spatial Patterns of NDVI Variation over Indonesia and their Relationship to ENSO Warm Events during the Period 1982-2006. Journal of Climate, 22, 6612-6623. DOI: 10.1175/2009JCLI2460.1

Hatfield, J.L. (2008). Application of Spectral Remote Sensing for Agronomic Decisions. Agronomy Journal, 100(3), 117131. DOI: 10.2134 /agronj2006.0370c

Jiménez, C.A., Vargas, V.T., Salinas, W.E., Aguirre, M., \& Rodríguez, D. (2004). Aptitud agroecológica para el cultivo de la caña de azúcar en el sur de Tamaulipas, México. Investigaciones Geográficas, Boletín del Instituto de Geografía, UNAM, 53, 58-74.

Kundu, A., Denis, D.M., Patel, N.R., \& Dutta, D. (2018). A Geospatial study for analysing temporal responses of NDVI to rainfall. Singapure Journal of Tropical Geography, 39, 107-116. DOI: $10.1111 /$ sjtg.12217

Lobo D., Cortez, A., Rodríguez, M.F., Ovalles, F., Rey, J.C., Gabriels, D., \& Parra, R.M. (2010). Análisis de la agresividad y concentración de las precipitaciones en Venezuela. I. Región de los llanos. Bioagro, 22(3), 169-176.

Lozano, Z., Lobo, D., \& Pla, S.I. (2002). Susceptibilidad a la degradación física de alfisoles de los llanos centrales y occidentales de Venezuela. Revista de la Facultad de Agronomía, 28, 41-57.
Olivares, B. (2012). Características agroclimáticas de la Mesa de Guanipa y requerimientos de clima y suelo de la planta de soya. En M. E. Martin, A. V. Parra, \& M. González (Coord.), El cultivo de la Soya en la Mesa de Guanipa (pp. 05-21). Caracas: MAT Editorial.

Olivares, B., Cortez, A., Lobo, D., Parra, R., Rey, J.C., \& Rodríguez, M.F. (2016b). Estudio de la Sequía Meteorológica en Localidades de los Llanos de Venezuela Mediante el Índice de Precipitación Estandarizado. Revista Acta Nova, 7(3), 266-283.

Olivares, B., Cortez, A., Muñetones, A., \& Casana, S. (2016c). Strategic Elements of Organizational Knowledge Management for Innovation. Case: Agrometeorology Network. Revista Digital de Investigación en Docencia Universitaria, 10(1), 68-81. DOI: 10.19083/ridu.10.446

Olivares, B., Cortez, A., Parra, R., Lobo, D., Rodríguez, M.F., \& Rey, J.C. (2017c). Evaluation of agricultural vulnerability to drought weather in different locations of Venezuela. Revista de la Facultad de Agronomía (LUZ), 34(1), 103-129.

Olivares, B., Cortez, A., Rodríguez, M.F., Rey, J.C., \& Lobo, D. (2016a). Desarrollo del sistema de información de la red de pluviómetros alternativos en medios rurales. Caso: Anzoátegui, Venezuela. Acta Universitaria, 26(4), 65-76. DOI: 10.15174/au.2016.961

Olivares, B., \& Franco, E. (2015). Diagnostico agrosocial de la comunidad indígena de Kashaama: Un estudio empírico en el estado Anzoátegui, Venezuela. Revista Científica Guillermo de Ockham, 13(1), 87-95. DOI: $10.21500 / 22563202.1691$

Olivares, B., Lobo, D., Cortez, A., Rodríguez, M.F., \& Rey, J.C. (2017a). Socio-economic characteristics and methods of agricultural production of indigenous community Kashaama, Anzoategui, Venezuela. Revista de la Facultad de Agronomía (LUZ), 34(2), 187-215.

Olivares, B., Parra, R., \& Cortez, A. (2017b). Caracterización de los patrones de precipitación en el estado Anzoátegui, Venezuela. Ería, 3(3), 353-365. DOI: 10.17811/ er.3.2017.353-365

Olivares B., Rodríguez, M., Cortez, A., Rey, J.C., \& Lobo, D. (2015). Caracterización físico natural de la comunidad indígena de Kashaama con fines de manejo sostenible de la tierra. Acta Nova, 7(2), 141-162.

Olivares, B., Torrealba, J., \& Caraballo, L. (2013). Variability of the precipitation regime in the period 1990-2009 in the location of El Tigre, Anzoátegui state, Venezuela. Revista de la Facultad de Agronomía (LUZ), 30(1), 19-32.

Olivares, B., \&Zingaretti, M.L. (2018). Análisis de la sequía meteorológica en cuatro localidades agrícolas de Venezuela mediante la combinación de métodos multivariados. UNED Research Journal, 10(1), 181-192.

Paredes, F., La Cruz, F., \& Guevara, E. (2014). Análisis regional de frecuencia de las sequías meteorológicas en la principal región cerealera de Venezuela. Bioagro, 26(1), 21-28. 
Peng, D., Zhang, B., Liu, L., Fang, H. Chen, D., Hu, Y., \& Liu, L. (2012). Characteristics and drivers of global NDVI-based FPAR from 1982-2006. Global Biogeochemical Cycles, 26, 1-15. DOI: 10.1029/2011GB004060

Poveda, G., \& Salazar, L.F. (2004). Annual and interannual (ENSO) variability of spatial scaling properties of a vegetation index (NDVI) in Amazonia", Remote Sensing Environment, 93, 391-401. DOI: 10.1016/j.rse.2004.08.001

R Development Core Team. (2008). R: A Language and Environment for Statistical Computing. Vienna (Austria): R Foundation for Statistical Computing.

Rodríguez, M.F., Cortez, A., Olivares, B., Rey, J.C., Parra, R., \& Lobo, D. (2013). Análisis espacio temporal de la precipitación del estado Anzoátegui y sus alrededores. Agronomía Tropical, 63(1-2), 57-65.

Rouse, J.W., Haas, R.H., Schell, J.A., \& Deering, D.W. (1973). Monitoring vegetation system in the great plains with ERTS. $3^{\text {rd }}$ ERST Symposium, NASA, 1, 309-317.

Rouse, J.W., Haas, R.W., Schell, J.A., Deering, D.H., \& Harían, J.C. (1974). Monitoring the vernal advancement andretrogadation (Greenwave effect) of natural vegetation (Progress Report RSC 1978-1). Greenbelt, Maryland, USA, NASA/ GSFCT.

Salinas, C.A., Martínez, R.O., \& Morales, M.V. (2017). Trend in the Normalized Diference Vegetation Index (NDVI) in the Southern Part of Baja California Peninsula. Investigaciones Geográficas, 94,82-90. DOI: 10.14350/ rig. 57214

Trejo, F.J.P., Barbosa, H.A., Peñaloza-Murillo, M.A., Moreno, M.A., \& Farías, A. (2016a). Intercomparison of improved satellite rainfall estimation with CHIRPS gridded product and rain gauge data over Venezuela. Atmósfera, 29(4), 323342. DOI: 10.20937/ATM.2016.29.04.04

Trejo, F.J.P., Barbosa, H.A., Ruiz, I.Q., \& Peñaloza-Murillo, M.A. (2016b). Meso scale oceanic atmospheric circulation patterns linked with severe and extensive droughts in Venezuela. Revista Brasileira de Meteorología, 31(4), 468489. DOI: 10.1590/0102-778631231420150070

Van Leeuwen, W.J.D., Hartield, K., Miranda, M., \& Meza, F.J. (2013). Trends and ENSO/AAO Driven Variability in NDVI Derived Productivity and Phenology alongside the Andes Mountains. Remote Sensing, 5, 1177-1203. DOI: $10.3390 /$ rs5031177

Yengoh, G.T., Dent, D., Olsson, L., Tengberg, A.E., \& Tucker, C.J. (Eds). (2015). Applications of NDVI for Land Degradation Assessment. In Use of the Normalized Difference Vegetation Index (NDVI) to Assess Land Degradation at Multiple Scales. Switzerland: SpringerBriefs in Environmental Science, Springer, Cham. DOI: 10,1007/978-3-319-24112-8_3 